TITLE:

\title{
Structure and generation mechanism of the peroxy-radical defect in amorphous silica
}

\section{$\operatorname{AUTHOR}(\mathrm{S})$ :}

Uchino, T; Takahashi, M; Yoko, T

\section{CITATION:}

Uchino, $T$...[et al]. Structure and generation mechanism of the peroxy-radical defect in amorphous silica. PHYSICAL REVIEW LETTERS 2001, 86(20): 4560-4563

\section{ISSUE DATE:}

2001-05-14

URL:

http://hdl.handle.net/2433/50393

RIGHT:

Copyright 2001 American Physical Society 


\title{
Structure and Generation Mechanism of the Peroxy-Radical Defect in Amorphous Silica
}

\author{
T. Uchino,* M. Takahashi, and T. Yoko \\ Institute for Chemical Research, Kyoto University, Uji, Kyoto 611-0011, Japan
}

(Received 16 January 2001)

\begin{abstract}
We provide a new model of the peroxy-radical defect in amorphous silica on the basis of quantumchemical calculations applied to clusters of atoms to model the defect. In this model, the ${ }^{29} \mathrm{Si}$ hyperfine splittings of the peroxy radical arise from a single silicon, in agreement with the previous experimental findings. Furthermore, we show that the present model of the peroxy radical is consistent with the diffusion-limited anneal mechanism of the $E_{\gamma}^{\prime}$ center, although our model of the $E_{\gamma}^{\prime}$ center is different from the conventional charged oxygen vacancy model.
\end{abstract}

In crystalline and amorphous silica ionizing radiation induces various types of structural defects [1,2], which cause degradation in $\mathrm{SiO}_{2}$-based electronic and optical materials, including metal-oxide-silicon integrated circuit devices and optical fiber waveguides. The major paramagnetic defect induced by ionizing radiation in amorphous silica $\left(a-\mathrm{SiO}_{2}\right)$ is the so-called " $E^{\prime}$ center [2]," which can be generically denoted by $\equiv \mathrm{Si} \bullet$ (where the three parallel lines represent three separate $\mathrm{Si}-\mathrm{O}$ bonds and the dot denotes the unpaired electron). Previous electron paramagnetic resonance (EPR) studies on irradiated $a-\mathrm{SiO}_{2}$ have demonstrated that there are several distinguishable variants of the $E^{\prime}$ center in terms of their $g$ values [3]. These $E^{\prime}$-center variants are also distinguished by virtue of different annealing kinetics [1]. Among other variants of the $E^{\prime}$ center, the $E_{\gamma}^{\prime}$ center has attracted much attention because it is the most commonly found photoinduced defect and is stable even above $\sim 500 \mathrm{~K}$. Thus, the annealing behavior of the $E_{\gamma}^{\prime}$ center has been extensively investigated during the past decades [4-9], although its mechanism has not been thoroughly delineated.

The annealing of the $E_{\gamma}^{\prime}$ center in dry $a-\mathrm{SiO}_{2}$ can be classified into two temperature regions [5]. At temperatures above $\sim 700 \mathrm{~K}$, the annealing process is irreversible and is probably associated with an irreversible structural reorganization around the defect site. On the other hand, the annealing of the $E_{\gamma}^{\prime}$ center at $\sim 500 \mathrm{~K}$ appears to be related to the thermally activated diffusion of $\mathrm{O}_{2}$ molecules, followed by the formation of the peroxy radical (POR). Since the annealing behavior of the $E_{\gamma}^{\prime}$ centers and PORs is dependent on the concentration of dissolved $\mathrm{O}_{2}$ molecules in $a-\mathrm{SiO}_{2}$, the following reaction of the $E_{\gamma}^{\prime}$ center and POR has been shown to exist [5]:

$$
E_{\gamma}^{\prime}+\mathrm{O}_{2} \rightarrow \text { POR }
$$

Furthermore, Zhang et al. [6] have demonstrated from repeated irradiation-anneal experiments that the POR can release an $\mathrm{O}_{2}$ molecule to form the $E_{\gamma}^{\prime}$ center, meaning that the reaction (1) is reversible. It was also confirmed from ${ }^{29} \mathrm{Si}$ hyperfine structure that the POR is strongly bonded to only one silicon atom in the glass network [10]; that is, the radical can be described as $\equiv \mathrm{Si}-\mathrm{O}-\mathrm{O} \bullet$. Such PORs have been shown to exist on the silica surface as well [11]. The EPR signal of the surface POR is almost identical with that of the bulk POR [11], indicating that the bulk and surface PORs have the same structural origin.

The above diffusion-limited annealing behavior of the $E_{\gamma}^{\prime}$ center was predicted theoretically by Edwards and Fowler [12] by using clusters of atoms that model the local configuration of the defects in $a-\mathrm{SiO}_{2}$. Since it was generally believed that the $E_{\gamma}^{\prime}$ center in $a-\mathrm{SiO}_{2}$ is essentially identical with the $E_{1}^{\prime}$ center in $\alpha$-quartz [13], they first started from a charged oxygen vacancy $\left(\equiv \mathrm{Si} \bullet{ }^{+} \mathrm{Si} \equiv\right)$ in the $\alpha$-quartz structure, which is the well-defined model of the $E_{1}^{\prime}$ center [14]. A neutral $\mathrm{O}_{2}$ molecule was then placed between the two silicon atoms to obtain a possible configuration of the POR according to reaction (1); the two central oxygen atoms and the two adjacent silicon atoms were allowed to search for positions of minimum total energy. For normal $\mathrm{Si}-\mathrm{Si}$ separations in $\alpha$-quartz (between $\sim 3.05$ and $3.25 \AA$ ), Edwards and Fowler [12] found that the resultant POR configuration, termed the small peroxy radical (SPOR), is strongly bonded to two silicon atoms; one of the two oxygen atoms was involved in this bonding, forming a three-coordinated oxygen [see Fig. 1(a)]. Experimentally, however, there is a ${ }^{29} \mathrm{Si}$ hyperfine interaction with only one silicon atom [10] as mentioned earlier, indicating that the SPOR model cannot be applied to the observed microscopic structure of the POR in $a-\mathrm{SiO}_{2}$. Edwards and Fowler [12], hence, relaxed such geometrical constraints as derived from $\alpha$-quartz by moving the two silicon atoms in the defect away from each other to simulate larger oxygen vacancies. They found that very large $\mathrm{Si}-\mathrm{Si}$ separations (the final $\mathrm{Si}-\mathrm{Si}$ separation is $6.01 \AA$ ) will be needed to fulfill the POR configuration attached to a single silicon atom. To our knowledge, this model, in which the two silicon atoms are widely apart in their relaxed state $\left(\equiv \mathrm{Si}-\mathrm{O}-\mathrm{O} \bullet{ }^{+} \mathrm{Si} \equiv\right)$, is the only model that can account for the mechanism of the diffusionlimited annealing behavior of the $E_{\gamma}^{\prime}$ center.

However, can such large $\mathrm{Si}-\mathrm{Si}$ separations $(\sim 6 \AA)$ be achieved in the actual silica network indeed? According to 
(a)

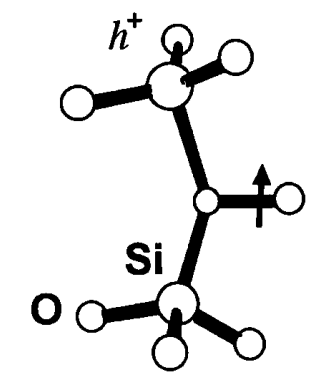

peroxy radical (SPOR)

(b)
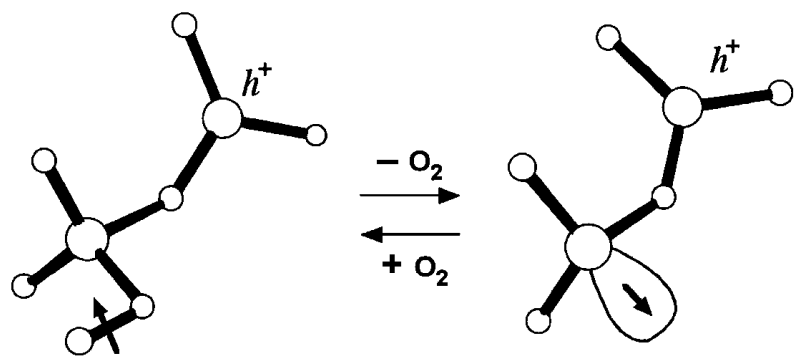

peroxy radical (LPOR)

FIG. 1. Models for the diffusion-limited interconversion of the peroxy-radical defect and the $E_{\gamma}^{\prime}$ center in $a-\mathrm{SiO}_{2}$ proposed in (a) Ref. [12] and (b) this study. In (a), i.e., the small peroxy radical (SPOR), the paramagnetic center is strongly bonded to two silicon atoms, whereas in (b), i.e., the large peroxy radical (LPOR), it interacts only with one silicon atom.

this model, the generation of the POR must require considerable structural reorganizations in the defect site, which may be inconsistent with the low temperature $(\sim 500 \mathrm{~K})$ annealing process described in reaction (1). Thus, we consider that the generation mechanism of the POR in $a-\mathrm{SiO}_{2}$ is still an open question. In this Letter, we, hence, propose an alternative structural model of the POR on the basis of quantum-chemical calculations, which will reasonably explain the interconversion of the $E_{\gamma}^{\prime}$ center and the POR along with the microscopic structures of these paramagnetic defects.

According to the EPR experiments [10], the paramagnetic $(\equiv \mathrm{Si}-\mathrm{O}-\mathrm{O} \bullet)$ and hole $\left({ }^{+} \mathrm{Si} \equiv\right)$ parts of the POR do not show a substantial hyperfine interaction, and, therefore, the conventional model requires very large $\mathrm{Si}-\mathrm{Si}$ separations to realize the observed POR structure. However, we here assume that the paramagnetic part and the hole part share the same one bridging oxygen atom, forming the configuration, $=\mathrm{Si}^{+}-\mathrm{O}-\mathrm{Si}-\mathrm{O}-\mathrm{O} \bullet$ [see also Fig. 1(b)]. In such a geometry, the constituent peroxy radical may be attached to only a single silicon atom without causing large $\mathrm{Si}-\mathrm{Si}$ separations because of the intervening bridging oxygen atom. We, therefore, performed a series of quantum-chemical calculations to investigate whether the above configuration is energetically feasible or not. Since the defect center proposed is bridged by a common oxygen atom and forms a larger POR configuration as compared with the SPOR, we will refer to this configuration as a "large peroxy radical (LPOR)." Figure 2 shows two different clusters, $\left(\mathrm{Si}_{6} \mathrm{O}_{19} \mathrm{H}_{12}\right)^{+}$and $\left(\mathrm{Si}_{8} \mathrm{O}_{23} \mathrm{H}_{12}\right)^{+}$, that model the local configuration of the LPOR. The $\mathrm{H}$ atoms in the clusters are used to saturate the dangling bonds of "surface" $\mathrm{O}$ atoms. In what follows, we will call these clusters model $1 \mathrm{a},\left[\left(\mathrm{Si}_{6} \mathrm{O}_{19} \mathrm{H}_{12}\right)^{+}\right]$, and model $1 \mathrm{~b},\left[\left(\mathrm{Si}_{8} \mathrm{O}_{23} \mathrm{H}_{12}\right)^{+}\right]$. The geometries of models 1a and $1 \mathrm{~b}$ were fully optimized at the density-functional theory (DFT) levels using the 6-31G $(d)$ basis set [15]. For the present DFT calculations, we used the B3LYP exchangecorrelation functional consisting of the Lee-Yang-Parr correlation functional [16] in conjunction with a hybrid exchange functional proposed by Becke [17]. All the quantum-chemical calculations in this work were carried out with the GAUSSIAN98 program [18].

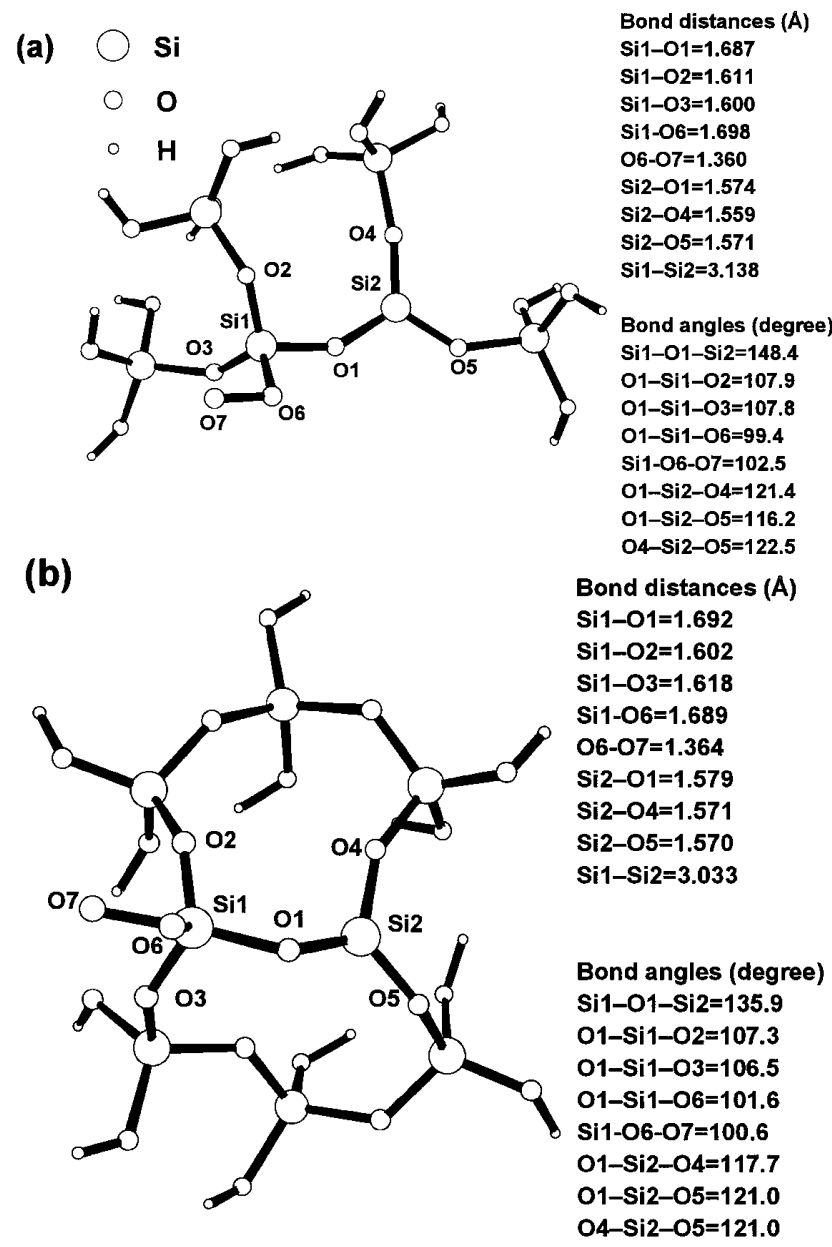

FIG. 2. Two different clusters of atoms that model the local configuration of the large peroxy radical: (a) model 1a $\left(\mathrm{Si}_{6} \mathrm{O}_{19} \mathrm{H}_{12}\right)^{+}$; (b) model $1 \mathrm{~b}\left(\mathrm{Si}_{8} \mathrm{O}_{23} \mathrm{H}_{12}\right)^{+}$. The geometries of the clusters were fully optimized at the B3LYP/6-31G $(d)$ level. Principal bond distances and bond angles are shown. 
TABLE I. Mulliken atomic charges, $q$, spin densities, $\rho$, and ${ }^{17} \mathrm{O}$ and ${ }^{29} \mathrm{Si}$ isotropic hyperfine coupling constants, $A$, for models $1 \mathrm{a}$ and $1 \mathrm{~b}$ calculated at the B3LYP/6-31G $(d)$ level along with the experimental spin-Hamiltonian parameters for peroxy radical defect.

\begin{tabular}{|c|c|c|c|c|c|c|c|c|}
\hline & $q$ & $\begin{array}{l}\text { Model 1a } \\
\qquad \rho\end{array}$ & $\begin{array}{c}A \\
(\mathrm{mT})\end{array}$ & $q$ & Model 1b & $\begin{array}{c}A \\
(\mathrm{mT})\end{array}$ & $\beta^{2}$ & $\begin{array}{c}\text { iment }^{\mathrm{f}} \\
A \\
(\mathrm{mT})\end{array}$ \\
\hline $\mathrm{O}^{\mathrm{a}}$ & -0.241 & $0.226^{\mathrm{b}}$ & -0.99 & -0.228 & $0.222^{\mathrm{d}}$ & -0.97 & 0.26 & -2.1 \\
\hline$O 7^{\mathrm{a}}$ & -0.091 & $0.781^{\mathrm{c}}$ & -1.93 & -0.080 & $0.787^{\mathrm{e}}$ & -1.94 & 0.74 & -4.0 \\
\hline $\mathrm{Si}^{\mathrm{a}}$ & 1.215 & -0.008 & 0.35 & 1.299 & -0.010 & 0.34 & & $0.36,^{\mathrm{g}} 0.42^{\mathrm{h}}$ \\
\hline $\mathrm{Si} 2^{\mathrm{a}}$ & 1.193 & 0.000 & 0.03 & 1.184 & 0.000 & 0.04 & & \\
\hline
\end{tabular}

${ }^{\mathrm{a}}$ For atom labels, see Fig. 2 .

${ }^{\mathrm{b}} s$-type orbitals $1.9 \%, p$-type orbitals $97.3 \%$, and $d$-type orbitals $0.8 \%$.

${ }^{\mathrm{c}} s$-type orbitals $0.7 \%, p$-type orbitals $99.2 \%$, and $d$-type orbitals $0.1 \%$.

${ }^{\mathrm{d}} s$-type orbitals $2.0 \%, p$-type orbitals $97.3 \%$, and $d$-type orbitals $0.7 \%$.

${ }^{\mathrm{e}} s$-type orbitals $0.7 \%, p$-type orbitals $99.2 \%$, and $d$-type orbitals $0.1 \%$.

${ }^{\text {f }}$ Refs. [3,10,19].

${ }^{\mathrm{g}} \mathrm{A}$ value for the $A_{1}\left({ }^{29} \mathrm{Si}\right)$ component.

${ }^{\mathrm{h}} \mathrm{A}$ value for the $A_{2}\left({ }^{29} \mathrm{Si}\right)$ component.

It has been found that, in the fully optimized structures of models $1 \mathrm{a}$ and $1 \mathrm{~b}$, the oxygens trapping the unpaired spin (O6 and O7, see Fig. 2) are bonded to only one silicon (Si1), in agreement with our expectation. Table I lists the Mulliken atomic charges, spin densities, and isotropic hyperfine parameters calculated for models $1 \mathrm{a}$ and $1 \mathrm{~b}$. We notice from Table I that the isotropic ${ }^{29} \mathrm{Si}$ hyperfine coupling $(\sim 0.35 \mathrm{mT})$ can be seen only for the silicon atom to which the peroxy oxygen atoms are attached (Si1). The present calculations, hence, reproduce the experimental EPR characteristics observed for POR [10]. It should also be worth mentioning the calculated spin densities on the peroxy-radical species in these models (see Table I). We have found that the unpaired spin has almost pure $p$ character, leading to the 22:78 distribution of spin density over the two oxygens. This calculated distribution of the unpaired spin agrees well with the experimental estimate (26:74) obtained from the ${ }^{17} \mathrm{O}$ hyperfine splittings [19]. Furthermore, the calculated ${ }^{17} \mathrm{O}$ isotropic hyperfine coupling of $\mathrm{O} 7$ is almost twice as large as that of O6, in accordance with the observed tendency. Thus, the present calculated results permit us to propose that the EPR signal associated with the POR in $a-\mathrm{SiO}_{2}$ results from the structure illustrated in Fig. 1(b), i.e., LPOR.

It is also interesting to investigate the defect structure after releasing the $\mathrm{O}_{2}$ molecule from the LPOR since such a defect center can be regarded as a precursor of POR. For this purpose, we intentionally removed the $\mathrm{O}_{2}$ mole- cule from models $1 \mathrm{a}$ and $1 \mathrm{~b}$ and reoptimized the geometries at the B3LYP/6-31G $(d)$ level by assuming a net positive charge $(+e)$. The resulting clusters will be termed model 2a, $\left[\left(\mathrm{Si}_{6} \mathrm{O}_{17} \mathrm{H}_{12}\right)^{+}\right]$, and model $2 \mathrm{~b},\left[\left(\mathrm{Si}_{8} \mathrm{O}_{21} \mathrm{H}_{12}\right)^{+}\right]$ (see Fig. 3). One sees from Fig. 3 that the bridged configuration is retained in models $2 \mathrm{a}$ and $2 \mathrm{~b}$ as in the case of models $1 \mathrm{a}$ and $1 \mathrm{~b}$. In the former cases, the $\equiv \mathrm{Si} \bullet$ and ${ }^{+} \mathrm{Si} \equiv$ units are bridged by a common oxygen atom, and, therefore, the defect configuration in models $2 a$ and $2 b$ can be referred to as a "bridged hole-trapping oxygen deficiency center (BHODC)." We have also found that the total energy of model 1a (model $1 \mathrm{~b}$ ) is lower than the sum of the total energy of model $2 \mathrm{a}$ (model $2 \mathrm{~b}$ ) and an $\mathrm{O}_{2}$ molecule by $2.27(2.22) \mathrm{eV}$ at the B3LYP/6-31G $(d)$ level of theory, indicating that the formation of LPOR from BHODC and $\mathrm{O}_{2}$ is energetically favorable. It is, hence, most likely that the BHODC can be a precursor of the POR [see also Fig. 1(b)], or more importantly, the BHODC can be identified with the $E_{\gamma}^{\prime}$ center according to reaction (1).

In a recent paper [20], we have shown that such a paramagnetic defect as seen in the BHODC is characterized by a localized unpaired spin in a single dangling $s p^{3}$ silicon orbital. The isotropic ${ }^{29} \mathrm{Si}$ hyperfine coupling of the paramagnetic Si in BHODC is calculated to be $41-44 \mathrm{mT}$, in agreement with the observed value for the $E_{\gamma}^{\prime}$ center in $a-\mathrm{SiO}_{2}$ (see also Table II). Thus, the present scenario for the generation of POR is consistent with our recent proposal [20] that the BHODC is a better model to describe the

TABLE II. Mulliken atomic charges, $q$, spin densities, $\rho$, and ${ }^{29} \mathrm{Si}$ isotropic hyperfine coupling constants, $A$, for models $2 \mathrm{a}$ and $2 \mathrm{~b}$ calculated at the B3LYP/6-31G(d) level along with the experimental hyperfine coupling constant reported for the $E_{\gamma}^{\prime}$ center.

\begin{tabular}{lccccccc}
\hline \hline & & Model 2a & & & Model 2b & \multicolumn{2}{c}{$\begin{array}{c}\text { Experiment }^{\text {b }} \\
A\end{array}$} \\
& $q$ & $\rho$ & $\begin{array}{c}A \\
(\mathrm{mT})\end{array}$ & $q$ & $\rho$ & -44.39 \\
$(\mathrm{mT})$
\end{tabular}

${ }^{\mathrm{a}}$ For atom labels, see Fig. 3.

${ }^{\mathrm{b}}$ Ref. [3]. 

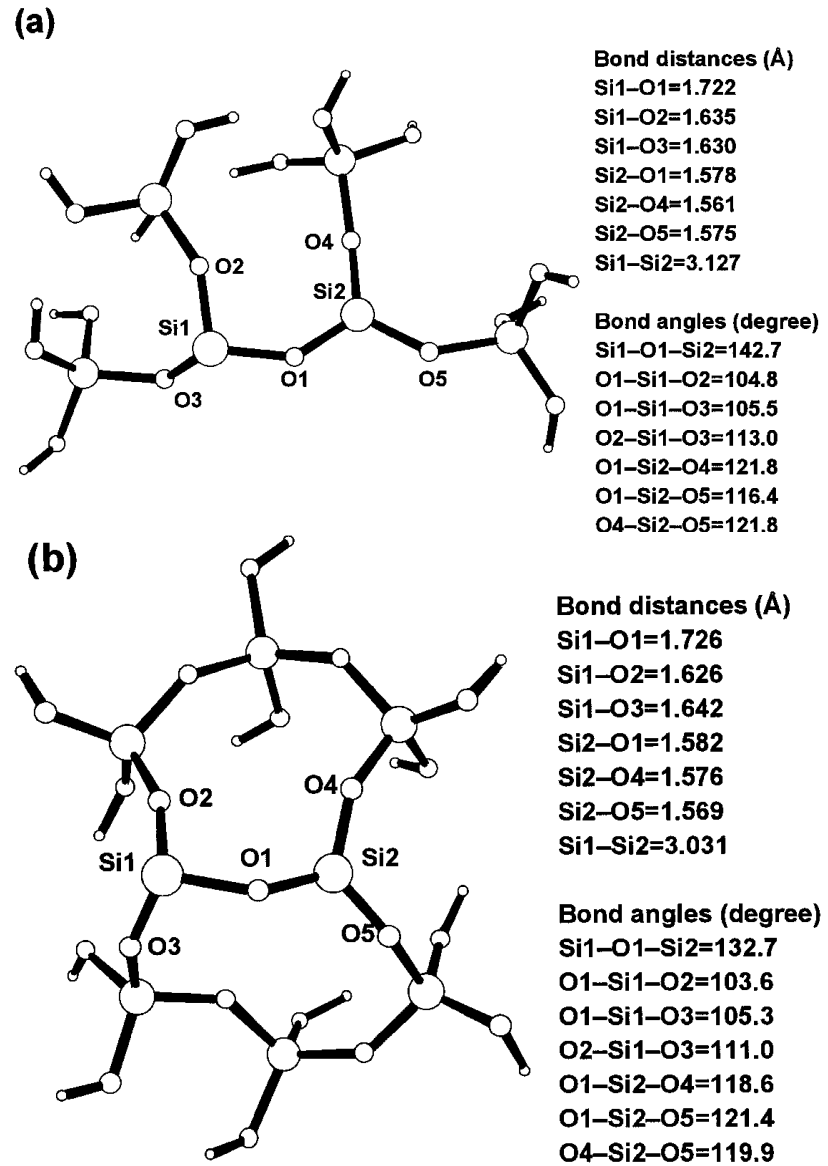

Bond distances (A)

Si1-01=1.726

Si1-02=1.626

Si $1-03=1.642$

$\mathrm{Si} 2-01=1.582$

Si2-04=1.576

Si2 $-05=1.569$

$\mathrm{Si1}-\mathrm{Si2}=\mathbf{3 . 0 3 1}$

Bond angles (degree)

Si1-01-Si2=132.7

O1-Si $1-02=103.6$

O1-Si1-03=105.3

O2-Si1-03=111.0

01-Si2-04=118.6

O1-Si2-05=121.4

O4-Si2-05=119.9

FIG. 3. Two different clusters of atoms that model the local configuration of the bridged hole-trapping oxygen deficiency center: (a) model $2 \mathrm{a}\left(\mathrm{Si}_{6} \mathrm{O}_{17} \mathrm{H}_{12}\right)^{+}$; (b) model $2 \mathrm{~b}\left(\mathrm{Si}_{8} \mathrm{O}_{21} \mathrm{H}_{12}\right)^{+}$. The geometries of the clusters were fully optimized at the $\mathrm{B} 3 \mathrm{LYP} / 6-31 \mathrm{G}(d)$ level. The initial configurations of models $2 \mathrm{a}$ and $2 \mathrm{~b}$ were obtained by removing an $\mathrm{O}_{2}$ molecule from models $1 \mathrm{a}$ and $1 \mathrm{~b}$, respectively. Principal bond distances and bond angles are shown.

microscopic structure of the $E_{\gamma}^{\prime}$ center in $a-\mathrm{SiO}_{2}$ than the conventional charged oxygen vacancy $\left(\equiv \mathrm{Si} \bullet{ }^{+} \mathrm{Si} \equiv\right)$ in an $\alpha$-quartz-like oxygen cage. The failure to observe the hypothetical defect represented in Fig. 1(a) also suggests that the $E_{\gamma}^{\prime}$ center in $a-\mathrm{SiO}_{2}$ should not be identical with the $E_{1}^{\prime}$ center in $\alpha$-quartz. Taking these things mentioned above into account, we conclude that the BHODC is a likely candidate for the $E_{\gamma}^{\prime}$ center as well as a precursor of the POR.

In summary, we have presented an unprecedented model of the POR in $a-\mathrm{SiO}_{2}$ on the basis of quantum-chemical calculations. The defect model, termed LPOR, can be viewed as a structural unit comprising the paramagnetic $(\equiv \mathrm{Si}-\mathrm{O}-\mathrm{O} \bullet)$ and hole $\left({ }^{+} \mathrm{Si} \equiv\right)$ parts that share one common bridging oxygen atom as shown in Fig. 1(b). Using DFT cluster calculations, we have demonstrated that the basic EPR characteristics observed for POR are rea- sonably reproduced by the present model. Furthermore, the present LPOR model gives a renewed insight into the microscopic origin of the $E_{\gamma}^{\prime}$ center along with its diffusion-limited annealing behavior. Thus, we consider that the LPOR model is highly promising in view of the annealing mechanism of the $E_{\gamma}^{\prime}$ center associated with $\mathrm{O}_{2}$ diffusion as well as the microscopic structure of the POR itself.

We thank the Supercomputer Laboratory, Institute for Chemical Research, Kyoto University, for providing the computer time to use a CRAY T-94/4128 supercomputer. We were supported by Grant No. 12CE2005 from the Ministry of Education, Culture, Sports, Science and Technology, Japan (Center of Excellence).

*Email address: uchino@scl.kyoto-u.ac.jp

[1] D. L. Griscom, J. Non-Cryst. Solids 73, 51 (1985).

[2] R. A. Weeks, J. Non-Cryst. Solids 179, 1 (1994).

[3] D. L. Griscom, in Glass-Science and Technology, edited by D. R. Uhlmann and N. J. Kreidl (Academic, London, 1990), Vol. 4B, pp. 151-251.

[4] M. Stapelbroek, D. L. Griscom, E. J. Friebele, and G. H. Sigel, Jr., J. Non-Cryst. Solids 32, 313 (1979).

[5] R.L. Pfeffer, in The Physics and Technology of Amorphous $\mathrm{SiO}_{2}$, edited by R. A. B. Devine (Plenum, New York, 1988), p. 181.

[6] L. Zhang, V. A. Mashkov, and R. G. Leisure, Phys. Rev. Lett. 74, 1605 (1995); Phys. Rev. B 53, 7182 (1996).

[7] D. L. Griscom, Nucl. Instrum. Methods Phys. Res., Sect. B 1, 481 (1984).

[8] E. J. Friebele, D. L. Griscom, and M. J. Marrone, J. NonCryst. Solids 71, 133 (1985).

[9] D. L. Griscom and M. Mizuguchi, J. Non-Cryst. Solids 239, 66 (1998).

[10] D. L. Griscom and E. J. Friebele, Phys. Rev. B 24, 4896 (1981).

[11] V.A. Radtsig and A. V. Bystrikov, Kinet. Catal. 19, 563 (1978).

[12] A. H. Edwards and W. B. Fowler, Phys. Rev. B 26, 6649 (1982).

[13] D. L. Griscom, Phys. Rev. B 22, 4192 (1980).

[14] F. J. Feigle, W. B. Fowler, and K. L. Yip, Solid State Commun. 14, 225 (1974).

[15] M. S. Gordon, Chem. Phys. Lett. 76, 163 (1980), and references therein.

[16] C. Lee, W. Yang, and R. G. Parr, Phys. Rev. B 37, 785 (1988).

[17] A. D. Becke, J. Chem. Phys. 88, 1053 (1988).

[18] M. J. Frisch et al., Gaussian 98, Revision A7 (Gaussian Inc., Pittsburgh, 1998).

[19] E. J. Friebele, D. L. Griscom, and M. Stapelbroek, Phys. Rev. Lett. 42, 1346 (1979).

[20] T. Uchino, M. Takahashi, and T. Yoko, Phys. Rev. B 62, 2983 (2000). 